Article

\title{
Improved Facial Expression Recognition Based on DWT Feature for Deep CNN
}

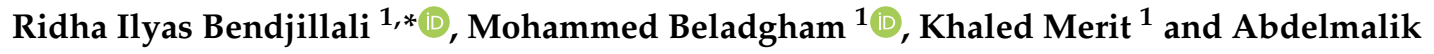 \\ Taleb-Ahmed ${ }^{2}$ \\ 1 Laboratory of TIT, Department of Electrical Engineering, Tahri Mohammed University, Bechar 08000, \\ Algeria; beladgham.tlm@gmail.com (M.B.); merit1984@gmail.com (K.M.) \\ 2 Laboratory of IEMN DOAE. UMR CNRS 852, University of Valenciennes, 59313 Valenciennes, France; \\ Abdelmalik.Taleb-Ahmed@univ-valenciennes.fr \\ * Correspondence: rayben43@gmail.com
}

Received: 18 January 2019; Accepted: 8 March 2019; Published: 15 March 2019

\begin{abstract}
Facial expression recognition (FER) has become one of the most important fields of research in pattern recognition. In this paper, we propose a method for the identification of facial expressions of people through their emotions. Being robust against illumination changes, this method combines four steps: Viola-Jones face detection algorithm, facial image enhancement using contrast limited adaptive histogram equalization (CLAHE) algorithm, the discrete wavelet transform (DWT), and deep convolutional neural network $(\mathrm{CNN})$. We have used Viola-Jones to locate the face and facial parts; the facial image is enhanced using CLAHE; then facial features extraction is done using DWT; and finally, the extracted features are used directly to train the CNN network, for the purpose of classifying the facial expressions. Our experimental work was performed on the $\mathrm{CK}+$ database and JAFFE face database. The results obtained using this network were $96.46 \%$ and $98.43 \%$, respectively.
\end{abstract}

Keywords: facial expression recognition (FER); deep convolutional neural network (deep CNN); discrete wavelet transform (DWT); contrast limited adaptive histogram equalization (CLAHE)

\section{Introduction}

Facial recognition is currently the most important biometric identification technology; this technique has many advantages, such as low cost and high reliability. Facial recognition has been used in several areas such as pattern recognition, computer vision, security, and cognitive science.

In recent years, facial expression recognition (FER) techniques aroused more and more interest on the part of the scientific community [1,2]. Facial expressions are an effective way in human and computer machine interaction and non-verbal interpersonal communications. It has many different applications in various fields such as security-surveillance, artificial intelligence, military and police services, and psychology, among others.

Facial expressions are classified into six basic categories; namely, anger, disgust, fear, sadness, happiness, and surprise-a neutral expression was also added to this group.

Facial expression recognition goes through three main steps. The first is face detection in the image. Its effectiveness has a direct influence on the performance of the FER system. The second important step of an FER system is facial features extraction, and the third and last step is expression classification.

In recent years, a wide variety of facial expression recognition methods have been proposed, such as elastic bunch graph matching (EBGM) [3], independent component analysis (ICA) [4], linear discriminant analysis (LDA) [5], principal component analysis (PCA) [6], scale invariant feature transform (SIFT) [7], and embedded hidden Markov models (EHMM) [8]. 
These methods have been used to improve the recognition rate and speed of facial expression recognition, but several challenges are confronted with regard to variations in the person's pose, changes in illumination, and so on.

Alternative methods are based on transforms such as Fourier transform (FT), short time FT (ST-FT), and discrete wavelet transform (DWT) [9]. Feature extraction based on the DWT method is very useful for FER with very low computational cost, which makes it an ideal tool for image processing and computer vision.

The main contributions of the proposed methodology are as follows: first, to develop a robust feature extraction approach; second, to improve the performance of the FER system and obtain a high recognition rate.

In this paper, we propose a model by applying the Viola-Jones face detection algorithm to detect faces, and to separate the faces from the rest of the parts considered non-faces. For our application, we have opted for different types of image enhancement algorithms to improve image contrast, the evaluation and judgment of each type in relation to the other is given by the calculation of the following parameters: absolute mean brightness error (AMBE) and peak signal to noise ratio (PSNR). Moreover, we employed the discrete wavelet transform (DWT) on face images to extract features.

Finally, the classification process will be done by deep learning through convolutional neural networks (CNN). Convolution neural networks are a type of artificial neural network that has been used in several areas such as classification, decision-making, and so on.

The rest of the paper is organized as follows. In Section 2, we deal briefly with the related work in this field; in Section 3, we describe the four steps of our facial expressions recognition system; we present our experimental results in Section 4; and finally, Section 5 concludes our work.

\section{Related Work}

In recent years, the scientific community has shown an increasing interest in the domain facial expressions; researchers have used several techniques in order to obtain a better representation of facial expressions, such as principal component analysis (PCA) and local binary patterns (LBP).

In this section, recent research on facial expression recognition (FER) using CNN that have a high degree of accuracy will be described.

In 2014, Liu et al., [10] used 3D-CNN for facial expression recognition (FER), and for localized action parts of the face, they used a deformable facial action part model.

In 2015, Peter Burkert et al., [11] used CNN for facial expression recognition (FER). The feature extraction proposed in this work is independent of any hand-craft. Dennis H. et al., [12] applied two CNN channels on facial image; the information is combined from the two channels to achieve $94.4 \%$ recognition accuracy.

In 2016, Cui, R. et al., [13] proposed an approach based on the CNN network that uses a set of outputs of three CNNs for classification.

In 2017, Nwosu, L. et al., [14] proposed an approach consisting of two steps: the Viola-Jones method for the detection of facial parts and deep CNN for feature extraction and classification. This method generates $97.71 \%$ and $95.72 \%$ recognition accuracy for JAFEE and CK+ datasets, respectively.

In 2018, Yang, B. et al., [15] proposed an FER method consisting of three steps: Viola-Jones face detection, local binary patterns (LBP) feature extraction, and weighted mixture deep neural network (WMDNN) based on double-channel facial images; the recognition accuracy for JAFEE and CK+ datasets is $92.21 \%$ and $97.02 \%$, respectively.

\section{The Proposed Methodology}

The automatic facial expression recognition system is performed in four main steps:

1. Face and facial parts detection. 
2. Facial image enhancement

3. Facial features extraction.

4. Expression classification.

In the case of a color image, the input image must be converted to grayscale. In what follows we will detail each step of the FER system, the proposed system is shown in Figure 1.

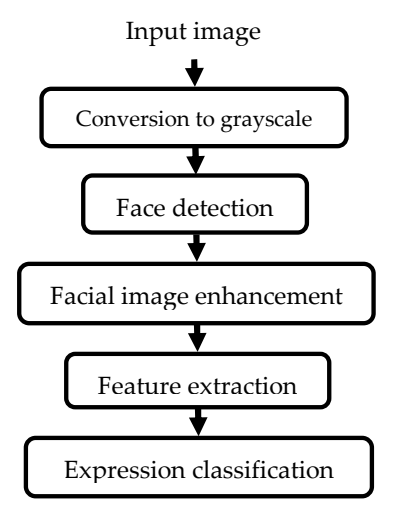

Figure 1. The steps of face recognition.

\subsection{Face Detection Using the Viola-Jones Algorithm}

The effectiveness of biometric systems based on face authentication essentially depends on the method used to locate the face in the image. In our method, we use the Viola-Jones algorithm to detect various parts of the human face such as the mouth, eyes, nose, nostrils, eyebrows, mouth, lips, and ears [16]. While several researchers are trying to reach an algorithm to detect the human face and its parts, the most effective algorithm one was proposed by Paul Viola and Michael Jones in 2001. This algorithm has been implemented in 'Matlab' using the vision Cascade Object Detector. There are three important techniques used by Viola-Jones for the detection of facial parts:

1. Haar-like features are digital image features of a rectangular type used in object recognition.

2. Ada boost is an artificial intelligence and machine learning method for face detection. The term 'boosted' determines a principle that brings together many algorithms that rely on sets of binary classifiers [17].

3. The third and last step is Cascade classifier, which can efficiently combine many features and determine the several filters on a resultant classifier. An example of the Viola-Jones algorithm is shown in Figure 2.

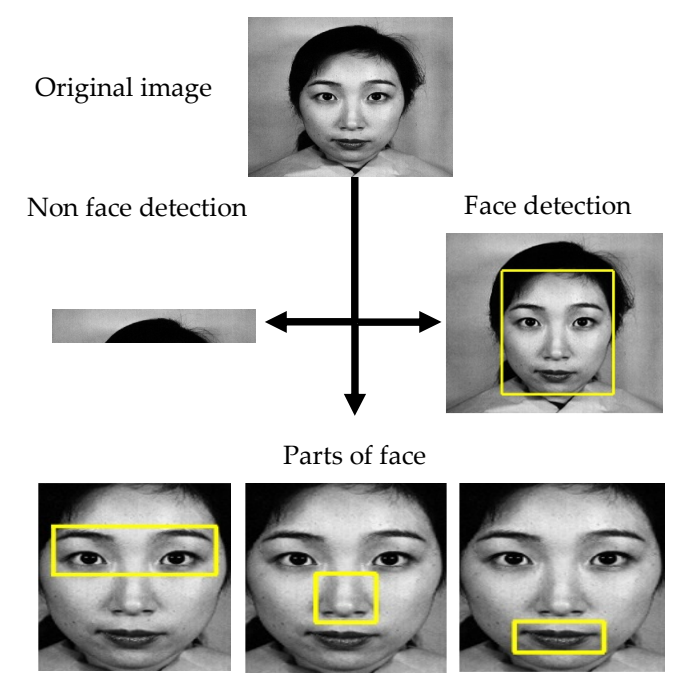

Figure 2. Detecting features of facial regions using the Viola-Jones algorithm. 


\subsection{Enhancement Techniques}

In this experiment, we have discussed a number of techniques for image enhancement such as the following.

\subsubsection{Histogram Equalization}

Histogram equalization is a method of adjusting the contrast of a digital image. It consists of applying a transform on each pixel of the image, and hence obtaining a new image from an independent operation on each of the pixels. This transform is constructed from the accumulated histogram of the original image [18].

The histogram equalization makes it possible to better distribute the intensities over the entire range of possible values by "spreading" the histogram. Equalization is interesting for images whose whole or only part is of low contrast (the set of pixels are of close intensity). The method is fast, easy to implement, and fully automatic.

Let $X$ be the input image, the intensity values in an image can be regarded as random variables that can have any value between $[0, L-1]$, the discrete gray levels in the dynamic range is $L$, and $X(i, j)$ represents the intensity of the image at spatial location $(i, j)$ that satisfies the condition $(i, j) \in\left\{X_{0}, X_{1}\right.$, $\left.\ldots, \mathrm{X}_{\mathrm{L}-1}\right\}$. The histogram ' $\mathrm{h}$ ' of the digital image is defined as the discrete function and is given by (1).

$$
\mathrm{h}\left(\mathrm{X}_{\mathrm{k}}\right)=\mathrm{n}_{\mathrm{k}}
$$

where

- $\quad \mathrm{X}_{\mathrm{k}}$ is the $\mathrm{k}_{\mathrm{th}}$ intensity level in the interval [0, $\left.\mathrm{L}-1\right]$.

- $\mathrm{n}_{\mathrm{k}}$ is the number of pixels in the input image.

The probability density function (PDF) is defined by (2).

$$
P\left(X_{k}\right)=\frac{h\left(X_{k}\right)}{M \times N}, \text { for } k=1,2, \ldots, L-1
$$

where $\mathrm{M} \times \mathrm{N}$ is the size of the image $\mathrm{X}$.

The cumulative distribution function (CDF) is obtained by (3).

$$
C\left(X_{k}\right)=\sum_{j=0}^{k} P\left(X_{j}\right), \text { for } k=1,2, \ldots, L-1
$$

HE is achieved by having a transform function $T\left(X_{k}\right)$, which can be defined as the (CDF) of a given (PDF) of gray-levels in a given image, which is defined as shown in (4).

$$
T\left(X_{k}\right)=(L-1) \times C\left(X_{k}\right), \text { for } k=1,2, \ldots, L-1
$$

The output image $Y$ denoted by $Y\{Y(i, j)\}=$ is defined in (5) below.

$$
Y=f(x)=\{f(x(i, j)) \mid \forall X(i, j) \in X\}
$$

This method successfully increases the global contrast of images, but it has several shortcomings such as the loss of some details in the image, some local areas become brighter than before, and it also fails to conserve the brightness of the image.

\subsubsection{Adaptive Histogram Equalization}

To avoid the drawbacks of the histogram equalization method discussed above, a modification of HE called the adaptive histogram equalization (AHE) can be used on such images for better results. 
In AHE, the input image is divided into small blocks called "tiles". Then, the histogram equalization method (AHE) is applied for each of these tiles using the CDF. It is, therefore, a local operation that can be enhanced simultaneously with all regions occupying different grayscale ranges, and enhancing the definitions of edges in each region of an image [19]. However, the AHE method has diverse drawbacks such as over amplifying the noise in the relatively homogeneous regions and a very high computational cost.

\subsubsection{Contrast Limited Adaptive Histogram Equalization}

The contrast limited adaptive histogram equalization (CLAHE) is a variant of adaptive histogram equalization (AHE). This method limits contrast amplification before computing the CDF by clipping the histogram at a predefined value, so as to overcome the problem of noise. The value at which the histogram is clipped, called clip limit depends on the normalization of the histogram and thereby on the size of the neighboring region [20].

\subsection{Extraction of Facial Features by Discrete Wavelet Transform (DWT)}

The extraction of features such as the eyes, nose, and mouth is a pre-treatment step necessary for facial expression recognition. In this step, we applied the discrete wavelet transform.

The wavelet is a famous tool in image processing and computer vision, and has several applications, such as compression, detection, recognition, and so on. The discrete wavelets transform (DWT) has the ability to locate a signal in both time and frequency resolutions at the same time. DWT is considered as a new generation of the discrete Fourier transform (DFT) [21].

DWTdecomposes the signal at several bands or frequencies; it involves filters of DWT known as the 'wavelet filter' and 'scaling filter'. The wavelet filters are a high pass filter and low pass filter. The DWT performs on different mother wavelets such as Haar, Symlet, and Daubechies.

In image processing, 2D-DWT is employed to perform operations throughout the rows of original images by employing both the low pass filter (LPF) and high pass filter (HPF) simultaneously [22]. Then, it is down-sampled by a factor of 2 and a detailed part (high frequency) and approximation part (low frequency) are achieved.

A further operation is performed throughout image columns. Four sub-bands are generated at each decomposition level: an 'approximation' sub-band (LL), and three 'detail' sub-bands-vertical ( $\mathrm{LH})$, horizontal (HL), and diagonal detail (HH) (see Figure 3). We considered 'Symelt' wavelet as amother wavelet in our approach [23].

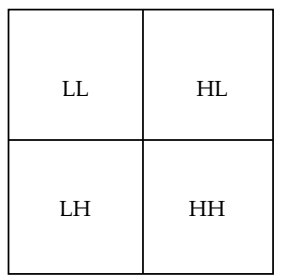

(a)

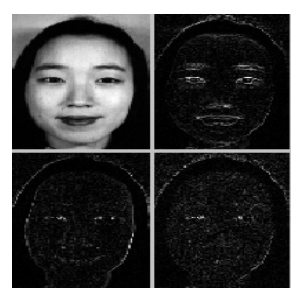

(c)

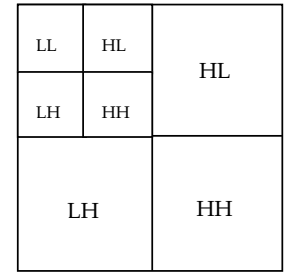

(b)

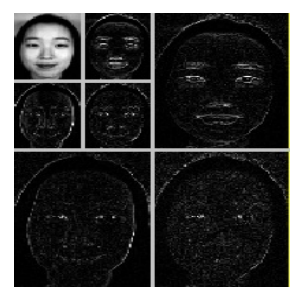

(d)

Figure 3. Multilevel wavelet decomposition consists of the following: (a) single level components specification, (b) two-level components specification, (c) discrete wavelet transform (DWT) single level, and (d) DWT two-level decomposition. LH—vertical; HL-horizontal; HH—diagonal detail. 


\subsection{Classification Using Deep Convolutional Neural Networks}

Convolutional neural networks are deep artificial neural networks, primarily used to classify images and group them based on similarity. CNNs are algorithms that can identify faces, character, human pose, tumors, street signs, and so on [24].

Through the use of discrete wavelet transform, features extraction of human face local texture was performed. The result is the input to the deep convolution neural network.

In this paper, as shown in Figure 4 below, we propose a network structure that contains three convolutions, two pooled layers, and one fully connected layer.

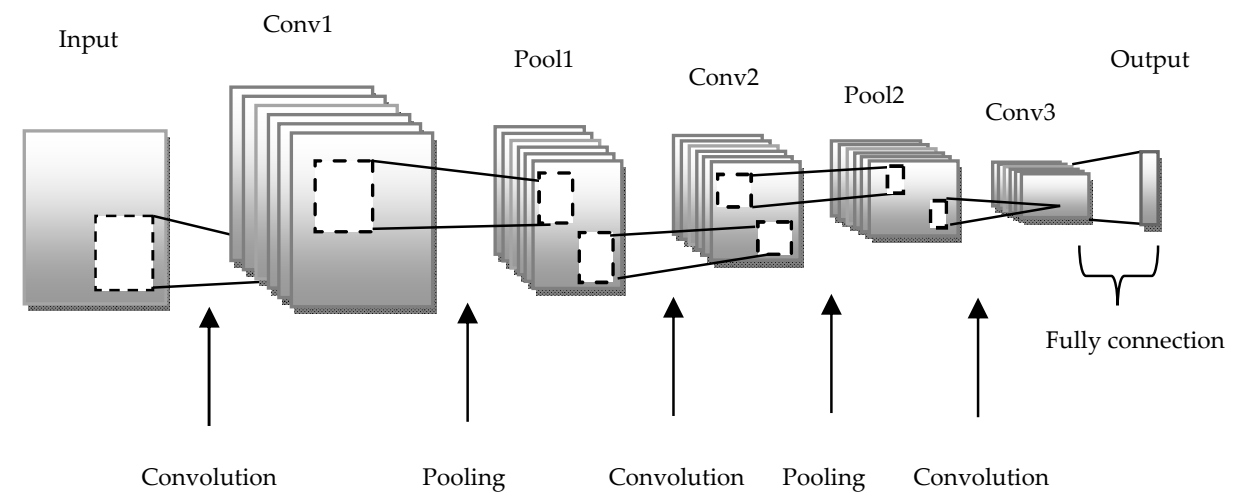

Figure 4. The proposed convolution network structure.

\subsubsection{Convolutional Layers}

One of the most important operations in the $\mathrm{CNN}$ is the convolutional layers (ConvL); CNN comprises one or more ConvL, and this latter is the basic building block that performs the core building block of a convolutional network that does most of the computational heavy lifting [25].

Like the traditional neural network, the input of each ConvL is the output of the upper layer, each of the feature graphs in the ConvL correspond to a kernel convolution of the same size and each of the feature maps of the ConvL is convoluted on a feature map of the previous layer [26], then bias is added after this process, after which the corresponding element finally obtained by activating the function is added.

Where the convolution kernel size of the first ConvL C1 is $5 \times 5$ and the size of the convolution kernel of the base layer C2 and C3 is $3 \times 3$, relative to $5 \times 5$, and for better results, the latter two convolutions use $3 \times 3$, because two $3 \times 3$ increase the network's non-linear capabilities, making the decision function more discriminative. However, if the first layer used is of $3 \times 3$, it will make the entire network model parameters too little, meaning a decrease in performance.

The mathematical expression of the layer [27] is as follows:

$$
x_{i}^{l}=f\left(\sum_{i \in M_{j}^{l-1}} x_{i}^{l-1} k_{i j}^{l}+b_{j}^{l}\right)
$$

where $l$ represents the layer, $f$ represents the activation function, $k$ is the convolution kernel, $b$ is the bias, and $M_{j}$ represents the feature map.

\subsubsection{Pooling}

The output feature maps obtained after the calculation of the ConvL are generally not greatly reduced in dimension. If the dimension does not change, a great amount of computation will be needed, and it will become very difficult to get a reasonable result with the network learning process [27]. 
The pooling layer is another important concept of CNNs that simplifies the output by performing nonlinear down-sampling, and reducing the number of parameters that the network needs to learn without changing the number of feature graphs. In this paper, the pooling layer is sampled with the maximum value. The sampling size is $2 \times 2$.

\subsubsection{Rectified Linear Unit (RELU)}

This is the most commonly used activation function in deep learning models, defined as the positive part of its argument, if the rectifier receives any negative input it will return to zero; it is defined as follows:

$$
f(x)=\max (0, x)
$$

\subsubsection{Full-Connected Layer}

For the network, after several convolutions and max-pooling layers, the high-level reasoning in the neural network is done via fully connected layers. All neurons in a fully connected layer have full connections to all activations in the previous layer, and these fully-connected layers form a multi-layer perceptron (MLP), which plays the role of a classifier.

\subsubsection{Output Layer}

The classifier layer is the output layer of the $\mathrm{CNN}$; the softmax regression classifier is used in this paper $[28,29]$. The softmax is a multi-classifier that has a strong non-linear classifying ability and is used at the last layer of the network; first, we enter the data $x$ for a given training.

Where the output category $y$ belongs to $\{1,2, \ldots, \mathrm{k}\}$, there are $\mathrm{k}$ classes in total; in this article we have set them to 10 . It is assumed that the input data $x$ are specified, the distribution probability of its class $y=i$ is as follows, $\theta i$ indicates the parameters to be fitted, e represents the base of the natural logarithm, and T represents the transpose. The meaning of $P(y=i \mid x ; \theta)$ is the probability that the input data $x$ corresponds to each class $i$ can take values 1 to $\mathrm{k}$.

$$
P\left(C_{j}=1 \mid x\right)=\frac{e^{w_{j}^{T} x}}{\sum_{i=1}^{k} e^{w_{j}^{T} x}}
$$

\section{Results and Discussion}

In this paper, the tests were performed on a personal computer (PC) 64 bit system with an I7 2.4 $\mathrm{GHz}$ processor and $8 \mathrm{~GB}$ of RAM using MATLAB R2018b.

\subsection{Performance Comparison of Enhancement Techniques}

The evaluation and judgment of each type of image enhancement technique based on histogram equalization are given by the calculation of the parameters absolute mean brightness error (AMBE), and peak signal to noise ratio (PSNR) [30].

\subsubsection{PSNR (Peak Signal to Noise Ratio)}

The metrics are the following:

$$
M S E=\frac{1}{M \times N} \sum_{i=1}^{M} \sum_{j=1}^{N}(I(i, j)-\hat{I}(i, j))^{2}
$$


where MSE is the mean square error, which requires two $M \times N$ grayscale images $I$ and $\hat{I}$. The PSNR is defined as follows:

$$
\text { PSNR }=10 \log _{10}\left(\frac{(255)^{2}}{M S E}\right)
$$

The great PSNR value estimates the degree of contrast enhancement. Table 1 summarizes the results obtained.

Table 1. The peak signal to noise ratio (PSNR) values for different algorithms. HE-histogram equalization; AHE—adaptive HE; CLAHE—contrast limited AHE.

\begin{tabular}{ccc}
\hline \multirow{2}{*}{ Algorithms } & \multicolumn{2}{c}{ Average PSNR } \\
\cline { 2 - 3 } & JAFFE Database & CK + Database \\
\hline HE & 15.11 & 11.46 \\
AHE & 25.86 & 17.08 \\
CLAHE & 41.00 & 31.49 \\
\hline
\end{tabular}

\subsubsection{AMBE (Absolute Mean Brightness Error)}

Another parameter is proposed to rate the performance in preserving image brightness; the absolute mean brightness error (AMBE) is defined by the following:

$$
\operatorname{AMBE}(x, y)=\left|X_{m}-Y_{m}\right|
$$

Or, $X_{m}$ and $Y_{m}$ are the mean intensities of the input and output image respectively [31].

On the contrary to PSNR, the least value of AMBE indicates better brightness preservation; Table 2 shows the results obtained.

Table 2. The absolute mean brightness error (AMBE) values for different algorithms.

\begin{tabular}{ccc}
\hline \multirow{2}{*}{ Algorithms } & \multicolumn{2}{c}{ Average PSNR } \\
\cline { 2 - 3 } & JAFFE Database & CK + Database \\
\hline HE & 16.49 & 44.48 \\
AHE & 8.69 & 15.11 \\
CLAHE & 4.42 & 5.92 \\
\hline
\end{tabular}

\subsection{The Visual Comparison}

The visual comparison of the facial image after enhancement is shown in this section (see Figures 5 and 6); the main goal is to judge if the enhanced facial image has a more natural appearance and is visually acceptable to the human eye. 


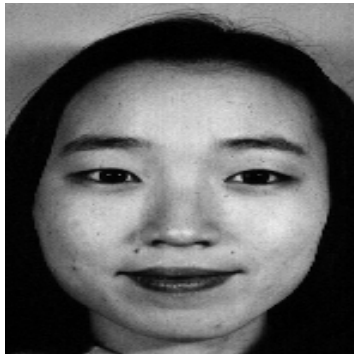

(a)

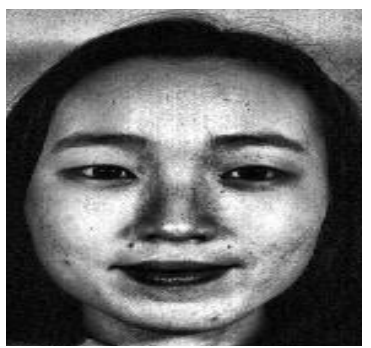

(c)

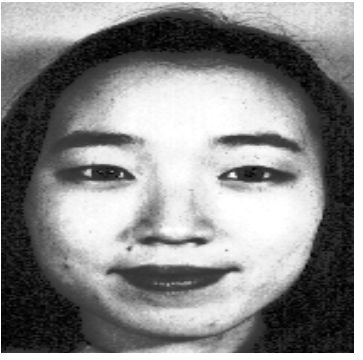

(b)

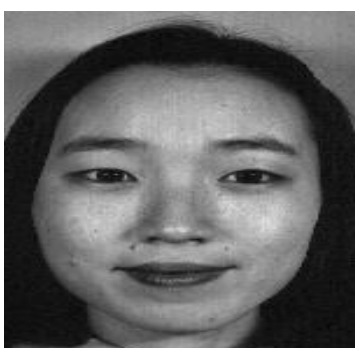

(d)

Figure 5. The results obtained from different algorithms on the JAFFE database: (a) original image, (b) histogram equalization (HE), (c) adaptive HE (AHE), and (d) contrast limited AHE (CLAHE).

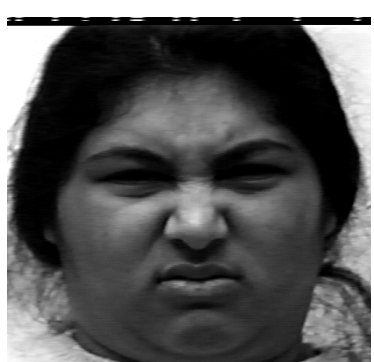

(a)

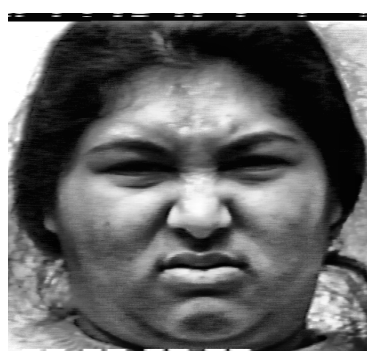

(c)

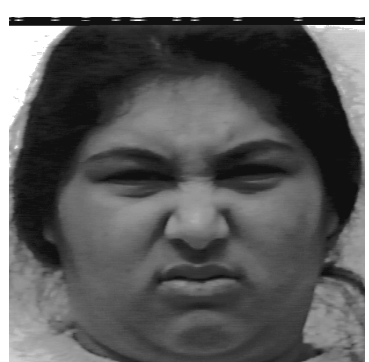

(b)

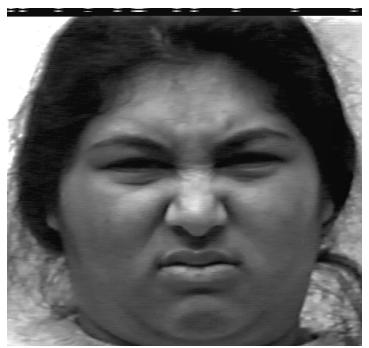

(d)

Figure 6. The result obtained using different algorithms on the $\mathrm{CK}+$ database: (a) original image, (b) HE, (c) AHE, and (d) CLAHE.

On the basis of visual observation, it can be concluded that the CLAHE technique provides better visual quality and a more natural appearance compared with other techniques.

After the visual observation, we focused on the impact of the clip-limit (CL) value and block size (Bs) of the CLAHE algorithm.

Firstly we fixed the Bs to [8 8] and varied the CL from 0.001 to 0.010 , after which we calculated the PSNR values of each variation.

The PSNR results of CLAHE are shown in Figure 7. 


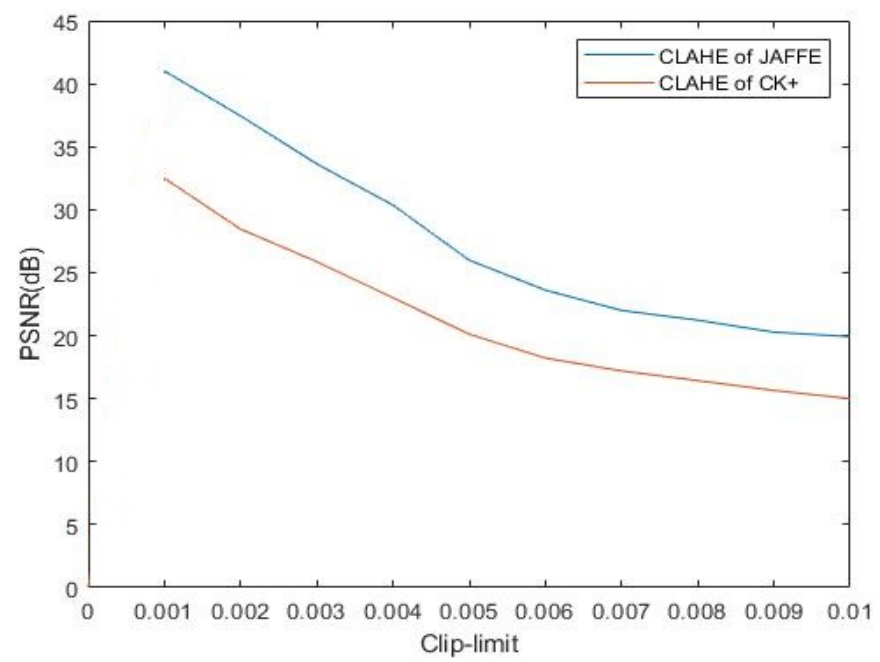

Figure 7. Peak signal to noise ratio (PSNR) values of each variation.

It can be observed from the figure that the CLAHE algorithm achieved the highest PSNR value at $\mathrm{CL}=0.001$ in the JAFEE and $\mathrm{CK}+$ databases.

Secondly, we fixed the clip limit value at 0.01 and varied the block size from [2 2] to [128 128], after which we calculated PSNR values of each variation (see Table 3).

Table 3. PSNR values of different block sizes.

\begin{tabular}{ccc}
\hline \multirow{2}{*}{ Block Size } & \multicolumn{2}{c}{ Average PSNR } \\
\cline { 2 - 3 } & JAFFE Database & CK+ Database \\
\hline $2 \times 2$ & 17.43 & 12.13 \\
$4 \times 4$ & 18.22 & 13.89 \\
$8 \times 8$ & 19.92 & 15.00 \\
$16 \times 16$ & 16.61 & 11.94 \\
$32 \times 32$ & 15.53 & 10.75 \\
$64 \times 64$ & 14.49 & 9.99 \\
$128 \times 128$ & 11.21 & 7.20 \\
\hline
\end{tabular}

It can be seen from the table that the PSNR achieved the highest value when the block size of [8 8] for JAFEE and CK+ databases was used.

\subsection{JAFFE Database}

The JAFFE database consists of 213 grayscale images of 10 Japanese female models; these images are almost frontal poses including 7 facial expression images; each image has a size of $256 \times 256$ [32]. The following illustration of the database is shown in Figure 8.

Firstly, we process the pictures from the JAFFE database as follows: the size of all the images was reduced to $64 \times 64$ pixels.

After that, contrast limited adaptive histogram equalization (CLAHE) was used for the contrast enhancement.

Finally, we used 149 images for training (about 70\% of the total) and 64 images for testing (about $30 \%$ of the total).

In Tables 4 and 5, N, A, D, F, H, Sa, and Su are used to represent seven basic expressions as neutral, anger, disgust, fear, happiness, sadness, and surprise, respectively. 

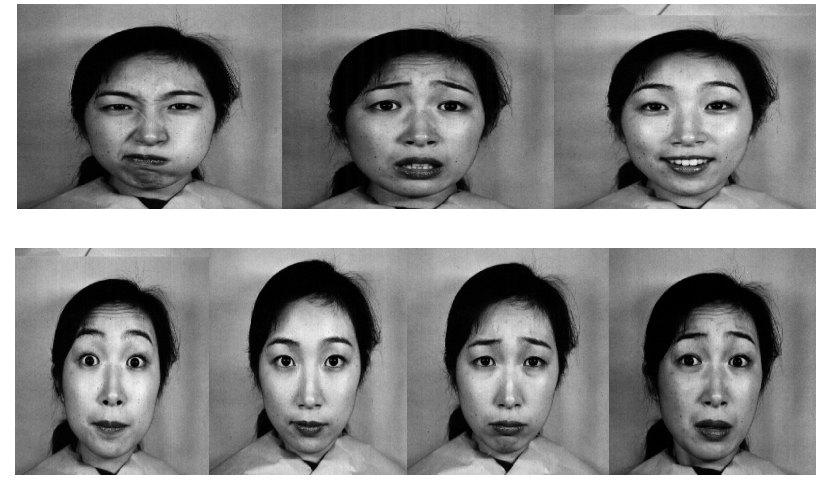

Figure 8. Partial image from the ORL database.

Table 4. The results obtained by the JAFFE database.

\begin{tabular}{cccccccc}
\hline & $\mathbf{N}$ & $\mathbf{A}$ & $\mathbf{D}$ & $\mathbf{F}$ & $\mathbf{S a}$ & $\mathbf{H}$ & $\mathbf{S u}$ \\
\hline $\mathbf{N}$ & 98.5 & 0.2 & 0.8 & 0.2 & 0.0 & 0.1 & 0.0 \\
$\mathbf{A}$ & 0.6 & 98.5 & 0.7 & 0.1 & 0.6 & 0.1 & 0.0 \\
$\mathbf{D}$ & 0.0 & 0.9 & 99.2 & 0.0 & 0.1 & 0.1 & 0.0 \\
$\mathbf{F}$ & 0.2 & 0.5 & 0.5 & 97.5 & 0.5 & 0.1 & 0.7 \\
$\mathbf{S}$ & 0.7 & 0.2 & 0.0 & 0.8 & 98.9 & 0.0 & 0.3 \\
$\mathbf{H}$ & 1.3 & 0.0 & 0.0 & 1.3 & 0.0 & 98.9 & 1.3 \\
$\mathbf{S}$ & 0.0 & 0.0 & 0.0 & 0.3 & 0.1 & 0.8 & 98.9 \\
\hline
\end{tabular}

The proposed method provided high recognition accuracy of $99.2 \%$ for disgust, $98.9 \%$ for surprise happiness and sadness; while anger, neutral, and fear had a high accuracy level but less than the previous facial expressions, with recognition accuracy of $98.5 \%-97.5 \%$ respectively. The JAFFE database achieved a recognition accuracy of $98.63 \%$.

\section{4. $C K+$ Database}

The $\mathrm{CK}+$ database consists of 593 images in total from 123 subjects that had a human facial emotion based on the subject's impression of each of the seven basic emotions [33].

The ages of participants are between 18 and 50,69\% of them are women, 81\% Euro-American, $13 \%$ Afro-American, and 6\% from other groups. Image sequences for frontal views and 30-degree views were digitized into either $640 \times 490$ or $640 \times 480$ pixel arrays. An illustration of the database is shown in Figure 9.
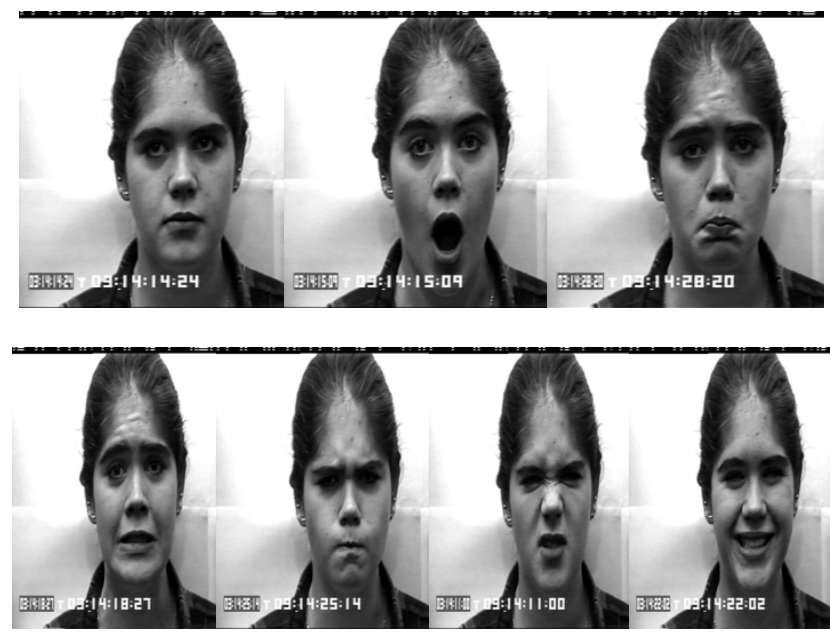

Figure 9. Partial image from the $\mathrm{CK}+$ database. 
Firstly, the pictures from the $\mathrm{CK}+$ database are processed as follows: the size of all the images was reduced to $64 \times 64$ pixels.

After that, for contrast enhancement, contrast limited adaptive histogram equalization (CLAHE) is used.

Finally, we used 415 images for training (about 70\% of the total) and 178 images for testing (about $30 \%$ of the total).

Table 5. The results obtained by the $\mathrm{CK}+$ database.

\begin{tabular}{cccccccc}
\hline & $\mathbf{N}$ & $\mathbf{A}$ & $\mathbf{D}$ & $\mathbf{F}$ & $\mathbf{S a}$ & $\mathbf{H}$ & $\mathbf{S u}$ \\
\hline $\mathbf{N}$ & 100 & 0.2 & 0.8 & 0.2 & 0.0 & 0.1 & 0.0 \\
$\mathbf{A}$ & 0.6 & 95.0 & 0.6 & 0.1 & 0.6 & 0.2 & 0.0 \\
$\mathbf{D}$ & 0.0 & 1.0 & 98.5 & 0.0 & 0.1 & 0.1 & 0.0 \\
$\mathbf{F}$ & 0.2 & 0.5 & 0.5 & 93.7 & 0.5 & 0.2 & 0.7 \\
$\mathbf{S}$ & 0.7 & 0.2 & 0.0 & 0.8 & 93.1 & 0.0 & 0.3 \\
$\mathbf{H}$ & 1.2 & 0.0 & 0.0 & 1.2 & 0.0 & 99.4 & 1.1 \\
$\mathbf{S}$ & 0.0 & 0.0 & 0.0 & 0.3 & 0.1 & 1.0 & 99.7 \\
\hline
\end{tabular}

The proposed method provided high recognition accuracy of $100 \%$ for neutral; $99.7 \%$ for surprised, $99.4 \%$ for happy, while angry disgust sad and fear had lower accuracy between $93.7 \%$ and $98.5 \%$. The $\mathrm{CK}+$ database gives a recognition accuracy of $97.05 \%$.

These results are satisfactory, but lower than those given using the JAFFE database; this is because the images in the $\mathrm{CK}+$ database were captured in a more difficult pose and under challenging lighting conditions.

\subsection{Results with and without Contrast Enhancement}

In order to demonstrate the effect of CLAHE on the recognition rate, we made a comparison between two methods; the first was used without the application of CLAHE.

The recognition rate results without the application of CLAHE enhancement algorithm for the JAFEE and CK+ database are shown in Table 6.

Table 6. Results without the application of CLAHE. RR-recognition rate.

\begin{tabular}{ccc}
\hline Expression & RR(\%) for JAFEE & RR(\%) for CK+ \\
\hline Neutral & 96.60 & 98.3 \\
Anger & 97.10 & 93.7 \\
Disgust & 97.30 & 96.5 \\
Fear & 96.17 & 92.4 \\
Happy & 96.63 & 90.8 \\
Sad & 96.90 & 97.3 \\
Surprise & 97.17 & 98.1 \\
\hline
\end{tabular}

The second method with the application of CLAHE provided the results shown in Table 7.

Table 7. Results with the application of CLAHE.

\begin{tabular}{ccc}
\hline Expression & RR(\%) for JAFEE & RR(\%) for CK+ \\
\hline Neutral & 98.5 & 100 \\
Anger & 98.5 & 95.0 \\
Disgust & 99.2 & 98.5 \\
Fear & 97.5 & 93.7 \\
Happiness & 98.9 & 93.1 \\
Sadness & 98.9 & 99.4 \\
Surprise & 98.9 & 99.7 \\
\hline
\end{tabular}


The comparison of the two methods with CLAHE and without CLAHE will show an improvement in the results, the recognition rate of the JAFFE database is improved by $1.9 \%$ for neutral, $1.4 \%$ for anger, $1.9 \%$ for disgust, $1.33 \%$ for fear, $2.27 \%$ for happiness, $2 \%$ for sadness, and $1.73 \%$ for surprise.

For the $\mathrm{CK}+$ database, the recognition rate has increased by $1.7 \%$ for neutral, $1.3 \%$ for anger, $2 \%$ for disgust, $1.3 \%$ for fear, $2.3 \%$ for happiness, $2.1 \%$ for sadness, and $1.6 \%$ for surprise.

\subsection{Comparison with Other Methods}

In order to prove the effectiveness of our approach, the average recognition accuracy is compared with other approaches for FER.

Tables 8 and 9 show the comparison of the recognition accuracy obtained with our approach and with other approaches for the JAFFE and CK+ databases.

Table 8. The comparison between different approaches and our approach for the JAFFE face database. $\mathrm{CNN}$ - convolutional neural network.

\begin{tabular}{cc}
\hline Approach & Recognition Rate \% \\
\hline SVM [34] & 95.60 \\
Gabor [35] & 93.30 \\
2-Channel CNN [12] & 94.40 \\
Deep CNN [14] & 97.71 \\
Normalization+ DL [36] & 88.73 \\
Viola-Jones+ CNN & 95.30 \\
Proposed Method & 98.63 \\
\hline
\end{tabular}

Table 9. The comparison between different approaches and our approach for the CK+ face database.CNN— convolutional neural network.

\begin{tabular}{cc}
\hline Approach & Recognition Rate \% \\
\hline SVM [37] & 95.10 \\
Gabor [35] & 90.62 \\
3D-CNN [38] & 95.00 \\
Deep CNN [14] & 95.72 \\
Normalization+ DL [36] & 93.68 \\
Viola-Jones+ CNN & 95.10 \\
Proposed Method & 97.05 \\
\hline
\end{tabular}

From the data in Tables 8 and 9, it is clear that our approach has achieved the highest recognition rate compared with $\mathrm{CNN}$ and the other approaches.

\subsection{Training Time}

In this section, we compared the training times of the $\mathrm{CNN}$ algorithm and the proposed algorithm in both databases. The comparison results are shown in Table 10.

Table 10. The training times comparison.

\begin{tabular}{ccc}
\hline Algorithm & JAFFE & CK+ \\
\hline CNN algorithm & $20.3 \mathrm{~s}$ & $33.3 \mathrm{~s}$ \\
Proposed algorithm & $15.7 \mathrm{~s}$ & $26 \mathrm{~s}$ \\
\hline
\end{tabular}

It can be seen from Table 10 above that the training time of the proposed algorithm is much shorter than that of the $\mathrm{CNN}$ algorithm; this means that our approach has a higher training speed and efficiency.

In short, the proposed algorithm greatly outperforms the traditional algorithm in terms of speed, recognition accuracy, and efficiency. 


\section{Conclusions}

This work presents a method of facial expressions recognition (FER) based on the Viola-Jones face detection algorithm, and facial image enhancement algorithms to improve image contrast. A comparative study of all these techniques has been presented. Through the results achieved after calculation of PSNR and AMBE parameters, we found that CLAHE outperforms all other techniques. Indeed, CLAHE clearly improves the contrast and brightness of the image more than the other enhancement techniques.

Then discrete wavelet transforms (DWT) and deep CNN are presented in this paper. Features extraction results of the face using DWT are the input to CNN network training, and the trained network is used for facial expressions recognition.

This network consists of three ConvL, two pooling layers, a fully-connected layer, and one softmax regression layer to classify and complete facial expressions recognition.

The results achieved on the JAFFEE and CK+ database confirm the effectiveness and robustness of our method. In experiments on the testing set of the JAFEE database and CK+ database, the expression recognition rate reaches up to $98.63 \%$ and $97.05 \%$, respectively.

Author Contributions: Conceptualization, R.I.B. and K.M.; methodology, R.I.B.; software, R.I.B.; validation, R.I.B., K.M. and M.B.; formal analysis, M.B.; investigation, A.T.-A.; resources, R.I.B.; data curation, R.I.B.; writing —original draft preparation, R.I.B.; writing—review and editing, R.I.B. and A.T.-A.; visualization, K.M.; supervision, M.B.; project administration, M.B. and A.T.-A.

Funding: This research received no external funding.

Acknowledgments: This work is supported by a research project about design and implementation of a surveillance system based on biometric systems for the detection and recognition of individuals and abnormal behaviors ( $\mathrm{N}^{\circ}$ A25N01UN080120180002).

Conflicts of Interest: The authors declare no conflict of interest.

\section{Abbreviations}

$\begin{array}{ll}\text { AHE } & \text { Adaptive Histogram Equalization } \\ \text { AMBE } & \text { Absolute Mean Brightness Error } \\ \text { CDF } & \text { Cumulative Distribution Function } \\ \text { ConvL } & \text { Convolutional Layers } \\ \text { CLAHE } & \text { Contrast Limited Adaptive Histogram Equalization } \\ \text { CNN } & \text { Convolutional Neural Network } \\ \text { DWT } & \text { Discrete Wavelet Transform } \\ \text { FER } & \text { Facial Expressions Recognition } \\ \text { HE } & \text { Histogram Equalization } \\ \text { LBP } & \text { Local Binary Patterns } \\ \text { PDF } & \text { Probability Density Function } \\ \text { PSNR } & \text { Peak Signal to Noise Ratio } \\ \text { RELU } & \text { Rectified Linear Unit } \\ \text { WMDNN } & \text { Weighted Mixture Deep Neural Network }\end{array}$

\section{References}

1. Yin, Y.; Li, Y.; Li, J. Face Festure Extraction Based on Principle Discriminant Information Analysis. In Proceedings of the IEEE International Conference on Automation and Logistics, Jinan, China, 18-21 August 2007; pp. 1580-1584.

2. Pantic, M.; Rothkrantz, L.J. Automatic analysis of facial expressions: The state of the art. IEEE Trans. Pattern Anal. Mach. Intell. 2000, 22, 1424-1445. [CrossRef]

3. Wiskott, L.; Fellous, J.M.; Kuiger, N.; Malsburg, C.V. Face Recognition by Elastic Bunch Graph Matching. IEEE Trans. Pattern Anal. Mach. Intell. 1997, 19, 775-779. [CrossRef]

4. Bartlett, M.S.; Movellan, J.R.; Sejnowski, S. Face Recognition by Independent Component Analysis. IEEE Trans. Neural Netw. 2002, 13, 1450-1464. [CrossRef] [PubMed] 
5. Belhumeur, P.N.; Hespanha, J.P.; Kriegman, D.J. Eigenfaces vs. Fisherfaces. IEEE Trans. Pattern Anal. Mach. Intell. 1997, 19, 711-720. [CrossRef]

6. Turk, M.; Pentland, A. Eigenfaces for Recognition. Cogn. Neurosci. 1991, 3, 71-86. [CrossRef] [PubMed]

7. Zhang, T.; Zheng, W.; Cui, Z.; Zong, Y.; Yan, J.; Yan, K. A deep neural network driven feature learning method for multi-view facial expression recognition. IEEE Trans. Multimed. 2016, 18, 2528-2536. [CrossRef]

8. Kim, D.J.; Chung, K.W.; Hong, K.S. Person authentication using face, teeth, and voice modalities for mobile device security. IEEE Trans. Consum. Electron. 2010, 56, 2678-2685. [CrossRef]

9. Wang, M.; Jiang, H.; Li, Y. Face Recognition based on DWT/DCT and SVM. In Proceedings of the International Conference on Computer Application and System Modeling (ICCASM 20ID), Taiyuan, China, 22-24 October 2010; pp. 507-510.

10. Liu, M.; Li, S.; Shan, S.; Wang, R.; Chen, X. Deeply learning deformable facial action parts model for dynamic expression analysis. In Computer Vision-ACCV 2014; Springer: Cham, Switzerland, 2014; pp. 143-157. [CrossRef]

11. Burkert, P.; Trier, F.; Afzal, M.Z.; Dengel, A.; Liwicki, M. DeXpression: Deep Convolutional Neural Network for Expression Recognition. arXiv, 2015; arXiv:1509.05371.

12. Hamester, D.; Barros, P.; Wermter, S. Face Expression Recognition with a 2-Channel Convolutional Neural Network. In Proceedings of the International Joint Conference on Neural Networks (IJCNN), Killarney, Ireland, 12-17 July 2015.

13. Cui, R.; Liu, M.; Liu, M. Facial Expression Recognition Based on Ensemble of Multiple CNNs. In Proceedings of the Biometric Recognition: 11th Chinese Conference, CCBR 2016, LNCS 9967, Chengdu, China, 14-16 October 2016; pp. 511-578.

14. Nwosu, L.; Wang, H.; Lu, J.; Unwal, I.; Yang, X.; Zhang, T. Deep Convolutional Neural Network for Facial Expression Recognition using Facial Parts. In Proceedings of the IEEE 15th International Conference on Dependable, Autonomic and Secure Computing, Orlando, FL, USA, 6-10 November 2017; pp. 1318-1321.

15. Yang, B.; Cao, J.; Ni, R.; Zhang, Y. Facial Expression Recognition Using Weighted Mixture Deep Neural Network Based on Double-Channel Facial Images. IEEE Access 2018, 6, 4630-4640. [CrossRef]

16. Viola, P.; Jones, M. Robust real-time object detection. In Proceedings of the Second International Work Shop on Statistical and Computational Theories of Vision, Vancouver, CA, Canada, 13 July 2001.

17. Vikram, K.; Padmavathi, S. Facial parts detection using Viola Jones Algorithm. In Proceedings of the 2017 4th International Conference on Advanced Computing and Communication Systems (ICACCS), Coimbatore, India, 6-7 January 2017.

18. Lee, P.-H.; Wu, S.-W.; Hung, Y.-P. Illumination compensation using oriented local histogram equalization and its application to face recognition. IEEE Trans. Image Process. 2012, 21, 4280-4289. [CrossRef] [PubMed]

19. Abdullah-Al-Wadud, M.; Kabir, M.H.; Dewan, M.A.A.; Chae, O. A Dynamic Histogram Equalization for Image Contrast Enhancement. IEEE Trans. Consum. Electron. 2007, 53, 593-600. [CrossRef]

20. Huang, L.; Zhao, W.; Wang, J.; Sun, Z. Combination of contrast limited adaptive histogram equalization and discrete wavelet transform for image enhancement. IET Image Process. 2015, 9, 908-915.

21. Dharani, P.; Vibhute, A.S. Face Recognition Using Wavelet Neural Network. Int. J. Adv. Res. Comput. Sci. Softw. Eng. 2017, 7. [CrossRef]

22. Benzaoui, A.; Boukrouche, A.; Doghmane, H.; Bourouba, H. Face recognition using 1dlbp, dwt and svm. In Proceedings of the 2015 3rd International Conference on Control, Engineering \& Information Technology (CEIT), Tlemcen, Algeria, 25-27 May 2015; pp. 1-6.

23. Dawoud, N.N.; Samir, B.B. Best Wavelet Function for Face Recognition Using Multi-Level Decomposition. In Proceedings of the IEEE International Conference on Research and Innovation in Information Systems, Nanjing, China, 24-25 September 2011.

24. Arel, D.; Rose, D.C.; Karnowski, T.P. Deep machine learning-A new frontier in artificial intelligence research [research frontier]. IEEE Comput. Intell. Mag. 2010, 5, 13-18. [CrossRef]

25. Coúkun, M.; Uçar, A.; Yildirim, Ö.; Demir, Y. Face Recognition Based on Convolutional Neural Network. In Proceedings of the International Conference on Modern Electrical and Energy Systems (MEES), Kremenchuk, Ukraine, 15-17 November 2017; pp. 376-379.

26. Wang, M.; Wang, Z.; Li, J. Deep Convolutional Neural Network Applies to Face Recognition in Small and Medium Databases. In Proceedings of the 4th International Conference on Systems and Informatics, Hangzhou, China, 11-13 November 2017; pp. 1368-1378. 
27. Yan, K.; Huang, S.; Song, Y.; Liu, W.; Fan, N. Face Recognition Based on Convolution Neural Network. In Proceedings of the 36th Chinese Control Conference, Dalian, China, 26-28 July 2017; pp. 4077-4081.

28. Zhou, N.; Wang, L.P. Class-dependent feature selection for face recognition. In Proceedings of the 15 th International Conference (ICONIP 2008), Auckland, New Zealand, 25-28 November 2008; pp. 551-558.

29. El Shafey, L.; Mccool, C.; Wallace, R.; Marcel, S. A Scalable Formulation of Probabilistic Linear Discriminant Analysis: Applied to Face Recognition. IEEE Trans. Pattern Anal. Mach. Intell. 2013, 35, 1788-1794. [CrossRef]

30. Sahu, S. Comparative Analysis of Image Enhancement Techniques for Ultrasound Liver Image. Int. J. Electr. Comput. Eng. 2012, 2, 792-797. [CrossRef]

31. Kim, M.; Chung, M.G. Recursively Separated and Weighted Histogram Equalization for Brightness Preservation and Contrast Enhancement. IEEE Trans. Consum. Electron. 2008, 54, 1389-1397. [CrossRef]

32. Lyons, M.J.; Akamatsu, S.; Kamachi, M.; Gyoba, J. Coding Facial Expressions with Gabor Wavelets. In Proceedings of the third IEEE International Conference on Automatic Face and Gesture Recognition, Nara, Japan, 14-16 April 1998; pp. 200-205.

33. Kanade, T.; Cohn, J.F.; Tian, Y. Comprehensive database for facial expression analysis. In Proceedings of the Fourth IEEE International Conference on Automatic Face and Gesture Recognition (FG'00), Grenoble, France, 26-30 March 2000; pp. 46-53.

34. Santiago, H.C.; Ren, T.; Cavalcanti, G.D.C. Facial expression Recognition based on Motion Estimation. In Proceedings of the 2016 International Joint Conference Neural Networks (IJCNN), Electronic Vancouver, BC, Canada, 24-29 July 2016.

35. Al-Sumaidaee, S.A.M. Facial Expression Recognition Using Local Gabor Gradient Code-Horizontal Diagonal Dedscriptor. In Proceedings of the 2nd IET International Conference on Intelligent Signal Processing 2015 (ISP), London, UK, 1-2 December 2015.

36. Lopes, A.T.; de Aguiar, E.; de Souza, A.F.; Oliveira-Santos, T. Facial expression recognition with convolutional neural networks: Coping with few data and the training sample order. Pattern Recognit. 2017, 61, 610-628. [CrossRef]

37. Shan, C.; Gong, S.; McOwan, P.W. Facial expression recognition based on local binary patterns: A comprehensive study. Image Vis. Comput. 2009, 27, 803-816. [CrossRef]

38. Byeon, Y.-H.; Kwak, K.-C. Facial expression recognition using $3 \mathrm{~d}$ convolutional neural network. Int. J. Adv. Comput. Sci. Appl. 2014, 5. [CrossRef] 